\title{
Both STAT1 and STAT3 are favourable prognostic determinants in colorectal carcinoma
}

\author{
C Gordziel $^{1}$, J Bratsch ${ }^{1}$, R Moriggl ${ }^{2}$, T Knösel $^{3,4}$ and K Friedrich ${ }^{*, 1}$
}

${ }^{1}$ Institute of Biochemistry II, University Hospital Jena, Jena, Germany; ${ }^{2}$ Ludwig Boltzmann Institute for Cancer Research, Vienna, Austria; ${ }^{3}$ Institute of Pathology, University Hospital Jena, Jena, Germany and ${ }^{4}$ Institute of Pathology, Ludwig-MaximiliansUniversity Munich, Munich, Germany

Background: Aberrant activities of Janus kinase (JAK)/signal transducer and activator of transcription (STAT) signalling pathways have been implicated in the development and spread of various cancer entities, among them colorectal carcinoma (CRC). Transcription factors STAT3 and STAT1, both downstream effectors of interleukin (IL)-6 and its receptor, are involved in growth and developmental control of CRC cells. Constituents of the signalling network around IL-6 and STAT activation are discussed as potential biomarkers and therapeutic targets in CRC.

Methods: By immunohistochemical analysis of a tissue microarray covering $>400 \mathrm{CRC}$ biopsies, the expression and activity status of STAT1, STAT3 as well as of IL-6 and the IL-6 receptor $\alpha$-chain was determined. The outcome was correlated with clinical information and patients' survival data. Colorectal carcinoma biopsies were also analysed for specific DNA-binding activity of STATs.

Results: Statistical analysis showed tendential associations between individual STATs, IL-6/IL-6 receptor- $\alpha$ and clinicopathological parameters. The study revealed a significant correlation of high STAT1 activity with longer patient overall survival. Surprisingly, strong STAT3 expression in surgical specimens was correlated with an increase in median overall survival by about 30 months. Statistical analysis revealed that high expression levels of STAT1 and STAT3 were associated. This finding was backed up by biochemical data that showed simultaneous STAT1 and STAT3 DNA-binding activity in randomly selected CRC biopsies.

Conclusion: By multivariate data analysis, we could show that STAT3 expression and activity constitutes an independent favourable prognostic marker for CRC.

As in many malignant tumours, the manifestation of a hyperproliferative and invasive phenotype of colorectal carcinoma (CRC) cells has been associated with abnormalities on the level of signal transduction. Signal transducer and activator of transcription (STAT) proteins as well as cytokine-driven Janus kinase (JAK)/STAT pathways have important roles in these processes as they frequently show alterations in their activities (Klampfer, 2008). Signal transducer and activator of transcription proteins are activated through tyrosine phosphorylation, mostly by cytokine receptor-associated JAKs. Subsequent dimerisation enables STATs to enter the nucleus and trigger gene expression by interacting with STAT cognate sequence in the DNA as well as with numerous regulatory proteins (Stark and Darnell, 2012). Various genes involved in potentially tumour-associated processes such as proliferation, cell differentiation and apoptosis are crucially influenced by STAT proteins (Bromberg et al, 1999; Kim and Lee, 2007; Murray, 2007; Regis et al, 2008; Sansone and Bromberg, 2012).

STAT3 activity is so far considered to be associated with elevated malignancy and invasive behaviour of CRC cells 
(Corvinus et al, 2005; Tsareva et al, 2007; Xiong et al, 2008, 2012; Zhuang et al, 2012). In CRC cell lines, STAT3 activation can upregulate the expression of matrix metalloproteinases, which in turn have the potential to favour cancer cell invasion and metastasis through proteolytic degradation of the extracellular matrix (Tsareva et al, 2007; Zugowski et al, 2011). However, recent findings from murine disease models indicate that STAT3 activity may also exert limiting effects on colonic carcinoma development. Interestingly, in Apc Min mice STAT3 was shown to suppress tumour cell invasiveness (Musteanu et al, 2010) and adenoma to carcinoma transition (Lee et al, 2012).

STAT1 is a signal mediator that controls functions of cell death in the context of interferon-dependent signalling, where it is involved in pro-apoptotic and anti-proliferative signalling pathways (Kim and Lee, 2007). STAT1 is generally regarded to have tumour suppressive functions and in some cancers its activity was shown to be associated with favourable prognosis (Widschwendter et al, 2002; Simpson et al, 2010).

Both STAT3 and STAT1 can become activated through the interleukin (IL)-6 receptor that consists of the IL-6 receptor $\alpha$-chain and the gp130 cytokine receptor chain (Heinrich et al, 2003). Interleukin- 6 is a pleiotropic cytokine and has been shown to exert tumour-promoting activities by enhancement of cell proliferation and suppression of apoptosis in various cancers. In CRC, increased levels of IL-6 in cancer tissue (Komoda et al, 1998) and serum (Chung and Chang, 2003; Chung et al, 2006) were described, as associated with advanced tumour stages and reduced survival. A soluble form of the IL- 6 receptor $\alpha$-chain is involved in IL-6dependent signalling in cancer and can confer IL- 6 responsiveness to cells that do not express membrane-bound IL-6R $\alpha$ by transsignalling (Rose-John et al, 2009).

The potential of individual and combined expression and/or activation of STAT3 and STAT1, and of their potential upstream activators (IL-6 and IL-6R $\alpha$ ) as predictors of survival in CRC has not been thoroughly investigated. This situation along with conflicting interpretations from sporadic clinical investigations in this field prompted us to perform a comprehensive study on the issue based on a tissue microarray covering $>400$ individual CRC tumour samples from a particularly homogeneous patient cohort.

\section{MATERIALS AND METHODS}

Patients, biopsies, tumour microarrays. In total, 414 tumour specimens (paraffin tissues) from patients with R0-resected primary colonic adenocarcinoma (CRC) of UICC stage II or III were included in this study. They had previously been employed to generate a CRC tissue microarray, as described (Knösel et al, 2012). Histological diagnosis was established according to the guidelines of the World Health Organisation. All patients had undergone surgical treatment at the Department of Surgery of the University Hospital Jena, and had not received adjuvant radiotherapy or chemotherapy before operation. Clinical parameters and follow-up data was available for all patients from the prospective tumour registry of the surgical clinic. Pathological findings (tumour size, tumour location and lymph node status) were obtained from board-certified pathological reports. The study was approved by the local ethics committee.

Immunohistochemistry. Immunohistological staining of TMAs was performed and evaluated essentially as described (Hamidov et al, 2011). Commercial antibodies employed were: anti-STAT1: Stat1 p84/p91 (M-22), Santa Cruz Biotechnology (Heidelberg, Germany), (sc-592, dilution 1:200); anti-STAT3: Stat3 (79D7) rabbit mAb, Cell Signaling Technology (Danvers, MA, USA) (number 4904, 1:400); anti-IL-6: anti-IL-6 antibody, Abcam (Cambridge, UK) (ab 6672, 1:600) and anti-IL-6R $\alpha$ : IL-6R $\alpha$ antibody (C-20), Santa Cruz (sc-661, dilution 1:200). Staining followed standard procedures. Briefly, the TMA slides were pretreated as described, incubated with the antibodies, followed by antibody detection via biotinylated anti-mouse secondary antibody and a biotin-streptavidin amplified detection system (Biogenex, San Ramon, CA, USA). Visualisation was done using a Fastred chromogen system (DAKO, Hamburg, Germany). For all antibodies, immunohistochemical staining was scored as negative (score 0 ), weak (score 1), moderate (score 2) or strong (score 3; Chen et al, 2003; Cui et al, 2011). Cytosolic localisation of staining was considered a measure for STAT expression whereas nuclear staining was considered indicative for STAT activity. Consequently, all TMAs were scored twice for nuclear and cytosolic STAT staining. For statistical evaluation, scores 0 and 1 were considered as 'low', whereas scores 2 and 3 were recorded as 'high'.

Statistical analysis. All data compilations were performed using the statistics software package SPSS (version 19.0; IBM, Armonk, NY, USA). $\chi^{2}$-test and Somer's d correlation coefficient were used to evaluate association between investigated parameters. $P$-values of $\leqslant 0.05$ were considered significant. Univariate survival analysis was carried out separately for each investigated parameter applying Kaplan-Meier estimate, the survival curves were compared and assessed using the log rank test. For multiple comparisons, the alpha value was adjusted applying the Bonferroni correction method. Multivariate survival analysis was performed using a proportional hazard model (Cox regression). Only parameters with $P$-values $<0.05$ in separate univariate survival analyses were included. A stepwise backward procedure then served to reduce the number of variables. $P$-values $<0.05$ were considered significant. All statistics were accredited by a biostatistician of the Institute of Medical Statistics, Computer Sciences and Documentation, Jena University Hospital.

Electrophoretic mobility shift assay. Whole-cell extracts were supplemented with tyrosine, serine and threonine phosphatase, protease inhibitors were prepared from snap-frozen biopsies, and protein concentration was determined by the Bradford protocol and adapted to equal levels. DNA-binding analysis was performed as described (Corvinus et al, 2005), employing the SIE-m67 STATbinding site from the human $\mathrm{c}$-fos promoter as a probe for the formation of DNA-STAT1/3 complexes. Binding reactions were performed by incubating 10000 c.p.m. of radiolabelled probe with $20 \mu \mathrm{g}$ of cell lysate for $30 \mathrm{~min}$ at room temperature. For supershift reactions of STAT-containing complexes, $2 \mu \mathrm{g}$ of antibodies to STAT1 (M-22 recognising the C-terminus; Santa Cruz Biotechnology) or STAT3 (C-20 or $\mathrm{H}-190$, recognising the $\mathrm{N}$-terminus or C-terminus; Santa Cruz Biotechnology) were added to the binding reactions. Samples were separated through $6 \%$ native polyacrylamide gels and complexes were visualised by autoradiography.

\section{RESULTS}

Correlation of clinicopathological parameters with patient survival. Table 1 shows the distribution of clinicopathological parameters among the patient cohort covered by the TMA used in this study. As shown in Table 1, the majority of tumour samples (about 90\%) showed advanced stages (stages 3 and 4) whereas the number of individual tumours showing high grading (grade 2 and 3 ) was almost equal to the number of low-grade tumours (grade 1). About $50 \%$ of the patients had already developed lymph node metastases. Vein invasion was scarcely found (about 18\% of cases). Univariate survival analysis, as expected, showed clear correlations of cancer-related clinicopathological parameters with the overall survival of patients represented by the TMA (Figure 1). Lymph node metastases and vein invasion significantly correlate with 
shorter patients survival $(P=0.003 / P=0.000$; Figure $1 \mathrm{~A}$ and $\mathrm{B})$. Lymph node metastases reduce the median survival by about 35 months (Figure 1A), vein invasion by about 70 months (Figure 1B). Shorter survival was tendentially associated with advanced tumour stages (T3/4), statistical significance, however, was not reached $(P=0.241)$ (Figure $1 C)$.

Table 1. Frequencies of different clinicopathological parameters within the patient cohort represented by the CRC tissue microarrays

\section{Number of cases}

$$
n=414
$$

Tumour staging (Union internationale contre le cancer (UICC) 2010)

\begin{tabular}{|l|r|r|}
\hline PT1 & 2 & 0.5 \\
PT2 & 41 & 9.9 \\
\hline PT3 & 320 & 77.3 \\
PT4 & 51 & 12.3 \\
\hline
\end{tabular}

Lymph node status (Union internationale contre le cancer (UICC) 2010)

\begin{tabular}{|l|r|r|}
\hline pN0 & 205 & 49.5 \\
pN1 & 130 & 31.4 \\
pN2 & 79 & 19.1 \\
\hline
\end{tabular}

\section{Lymph vessel invasion}

\begin{tabular}{|l|l|l|}
\hline L0 & 203 & 56.1 \\
\hline L1 & 159 & 43.9 \\
\hline
\end{tabular}

\section{Vein invasion}

\begin{tabular}{|l|r|r}
\hline V0 & 285 & 81.7 \\
V1 & 64 & 18.3 \\
\hline
\end{tabular}

\section{Clinical staging (UICC 2010)}

\begin{tabular}{|l|r|r}
\hline Stage I & 1 & 0.2 \\
Stage II & 204 & 49.3 \\
Stage III & 209 & 50.5 \\
\hline
\end{tabular}

\begin{tabular}{|l|r|r|}
\hline \multicolumn{3}{|c|}{ Grading } \\
\hline G1 & 203 & 56.1 \\
G2 & 159 & 43.9 \\
G3 & 0 & 0.0 \\
\hline
\end{tabular}

Abbreviation: $\mathrm{CRC}=$ colorectal carcinoma
Immunohistochemical analysis of CRC tissue microarrays for IL-6/STAT pathway proteins. TMAs were stained with antibodies to STAT1, STAT3, IL-6 and the IL-6 receptor $\alpha$-chain. To distinguish between STAT expression and STAT activation, staining for both STAT1 and STAT3 was separately determined in the cytosolic and in the nuclear compartment. Representative examples for different staining intensities and their respective scoring are shown in Figure 2. Results for the entire tumour collective and all antibodies are summarised in Figure 3. These data revealed a notable difference with regard to respective activities of STAT1 and STAT3. Although only 13\% of biopsies were entirely negative for activated (nuclear) STAT1 (score 0), almost half of the samples did not show even traces of nuclear STAT3. Another interesting observation was the relatively high fraction of samples with particularly strong expression of IL- 6 receptor- $\alpha$. On the basis of a binary classification of staining intensities ('low': covering scores 0 and 1, and 'high': scores 2 and 3, respectively), high expression (cytosolic localisation) of STAT1 was found in $35 \%$ of the tumour samples, high potential transcriptional activity (nuclear localisation) was observed in $48 \%$ of cases. STAT3 was highly expressed in $32 \%$ and highly activated (nuclear staining) in $30 \%$ of cases. High expression of IL- 6 was observed in only $10 \%$ of the biopsies, while roughly half of the samples showed high expression of the IL- 6 receptor $\alpha$-chain.

Correlation of patient survival with expression/activation of IL-6/STAT pathway proteins in CRC tissue. Figure 4 shows the correlation of staining results with patient overall survival by separate univariate analysis. STAT1 activation and, particularly interesting, also STAT3 expression were found significantly correlated with longer patients' overall survival $(P=0.010 /$ $P=0.020$; Figure $4 \mathrm{~A}$ and $\mathrm{D}$ ). The median survival of patients with high STAT1 activity was at least 30 months longer in comparison with patients with low tumour-related STAT1 activity. The median survival of patients with high STAT3 expression was increased by about 30 months at least in comparison with patients with low tumour-related STAT3 expression. Unlike these two parameters, STAT1 expression (cytosolic staining) and STAT3 activation (nuclear staining) were not significantly associated with clinical outcome. They showed, however, trends for a correlation with longer survival $(P=0.19 / P=0.16$; Figure $4 \mathrm{~B}$ and $\mathrm{C})$. This also applies for the expression of IL- 6 and the IL- 6 receptor $\alpha$-chain $(P=0.19 / P=0.26$; Figure $4 \mathrm{E}$ and $\mathrm{F})$.

We found no significant correlation of clinicopathological parameters with STAT1 or STAT3 protein expression or activation status. However, some clear trends could be observed, for example, a tendency for association of high cytosolic abundance of STAT3
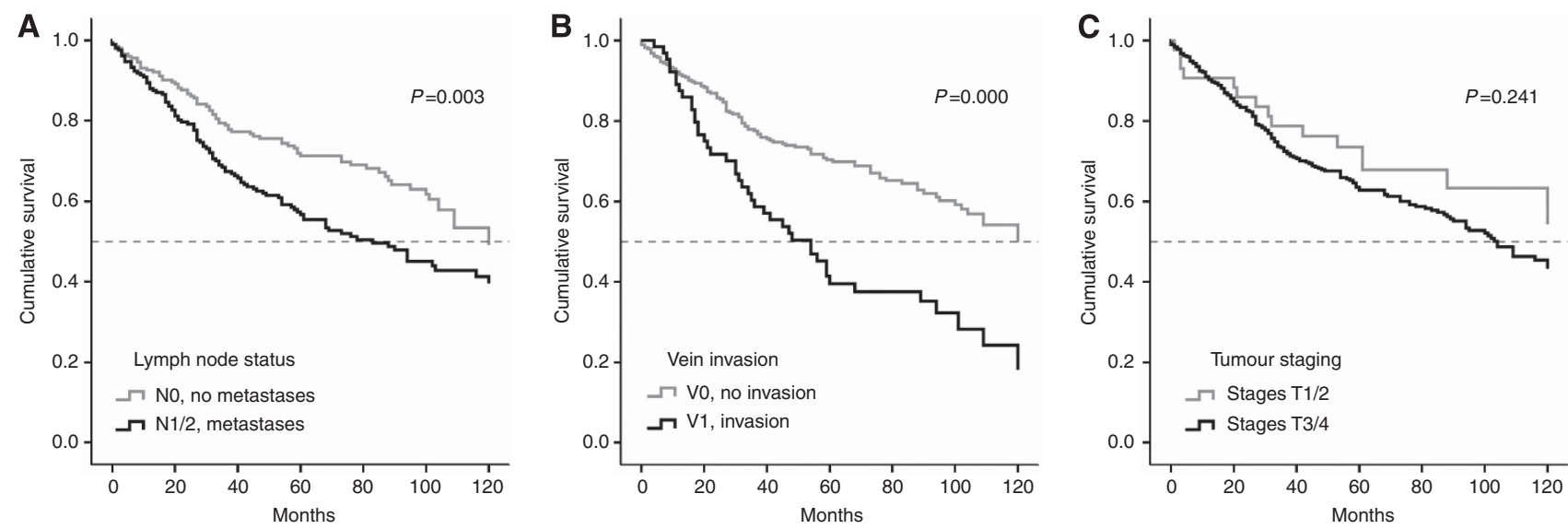

Figure 1. Univariate analysis of overall patients' survival in correlation with clinicopathological parameters. Patient survival related to (A) positive or negative lymph node metastasis state, (B) present or absent tumour-related vein invasion and (C) tumour stages 1/2 vs 3/4. 
A
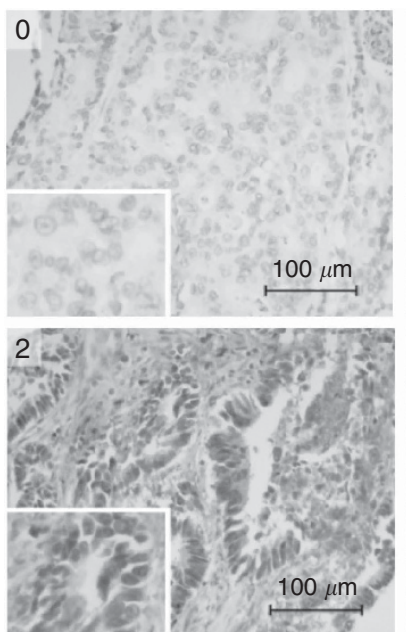

STAT1 nuclear

C
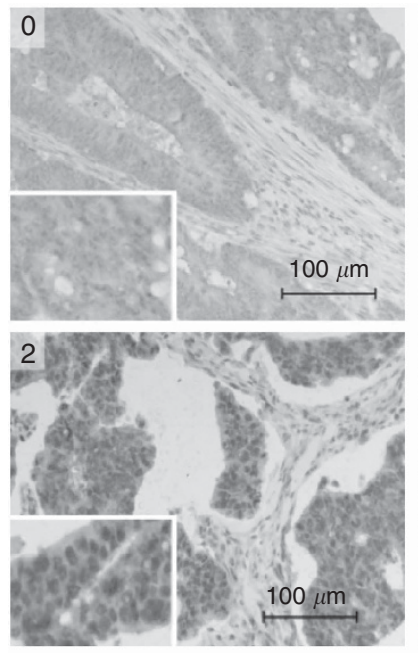

STAT3 nuclear

\section{E}
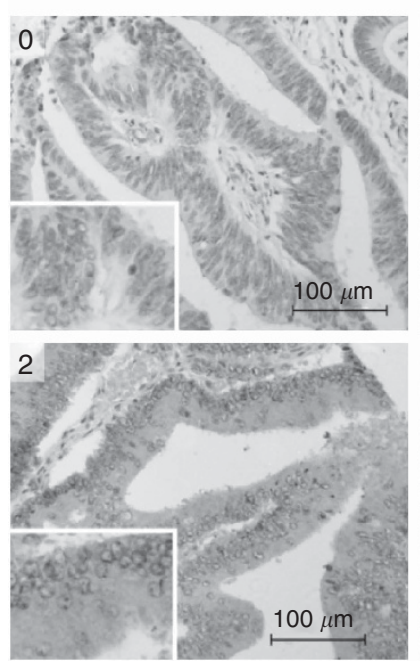

IL-6
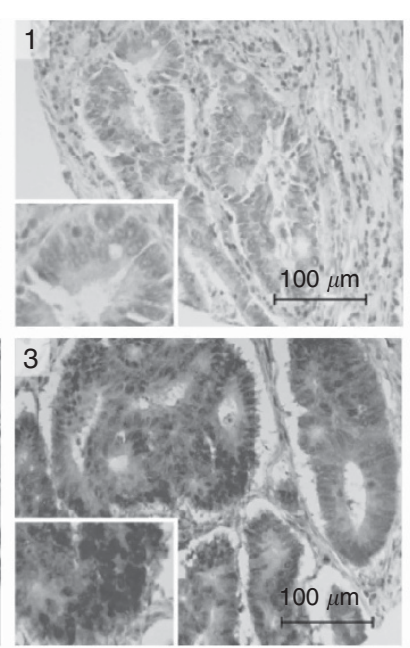

(10)
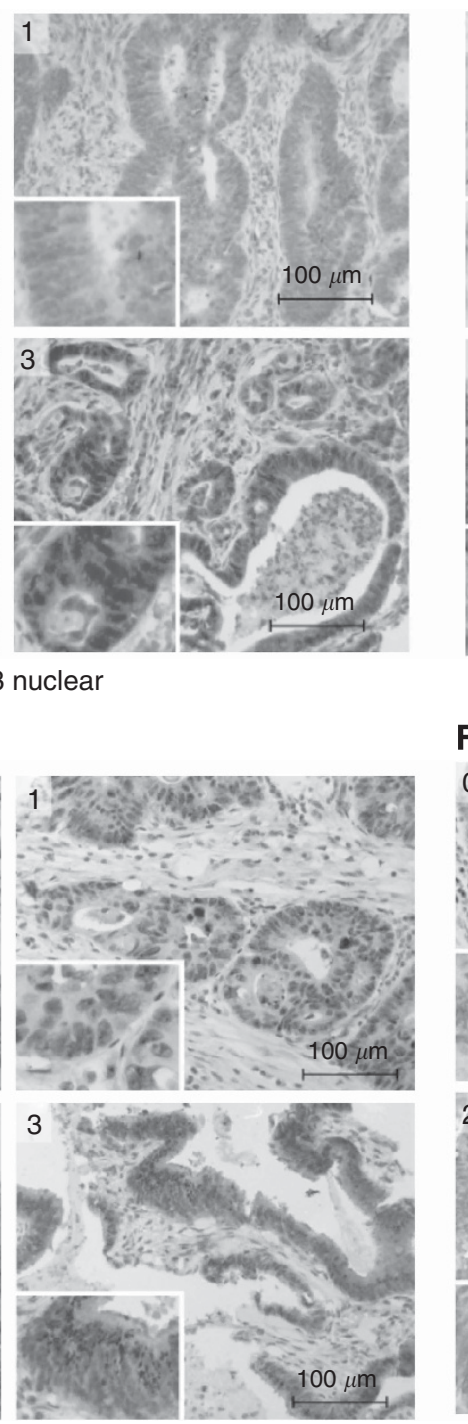

\section{B}
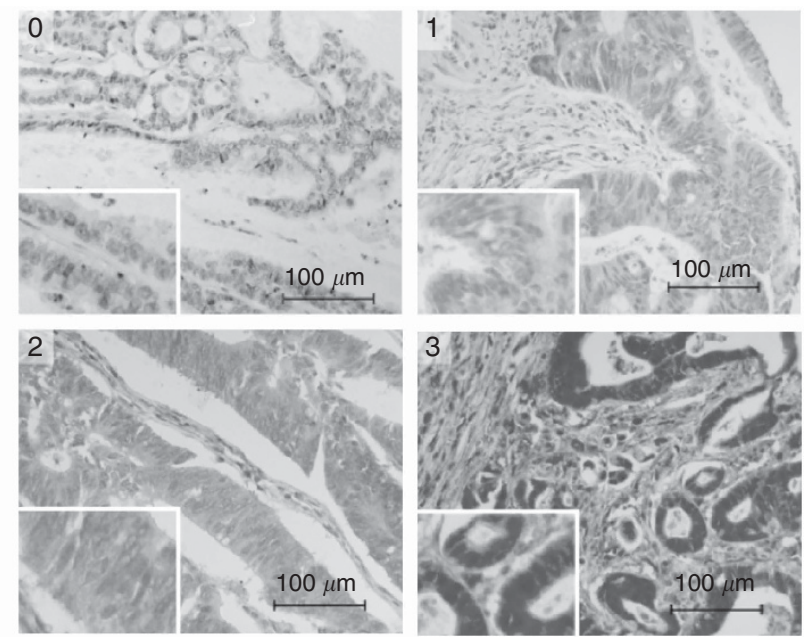

STAT1 cytosolic

D
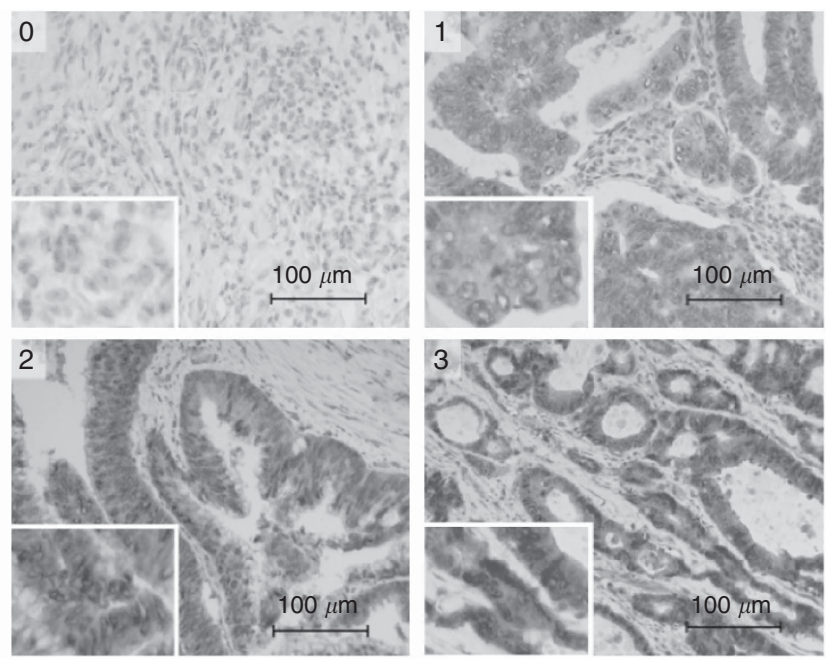

STAT3 cytosolic

\section{F}
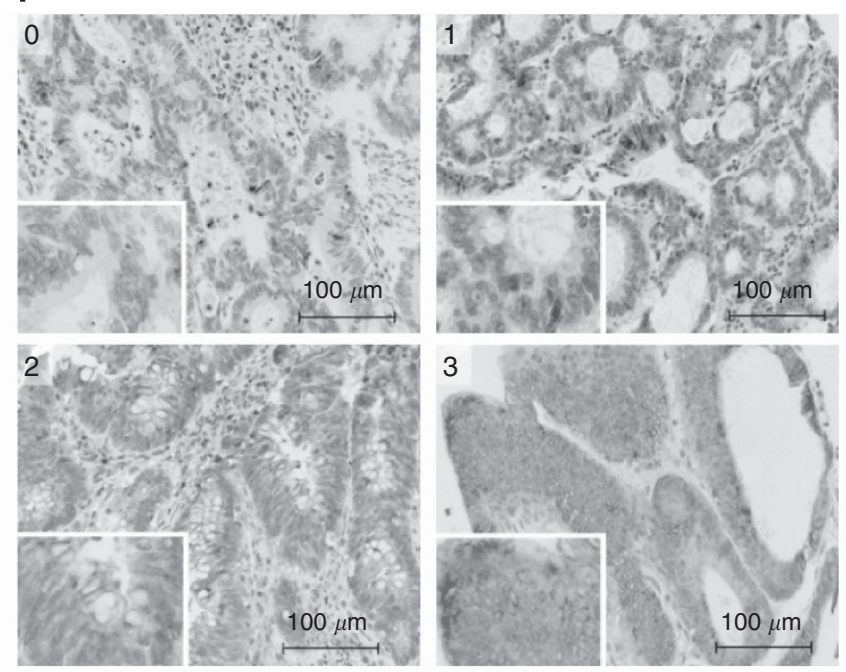

IL-6R $\alpha$

Figure 2. Immunohistochemical staining of CRC tissue microarrays for IL-6/STAT pathway proteins. Each panel shows representative examples for biopsies scored for staining intensity as 0 (negative), 1 (weak), 2 (moderate) and 3 (strong). (A) nuclear STAT1, (B) cytosolic STAT1, (C) nuclear STAT3, (D) cytosolic STAT3, (E) IL-6, (F) IL-6 receptor $\alpha$. 

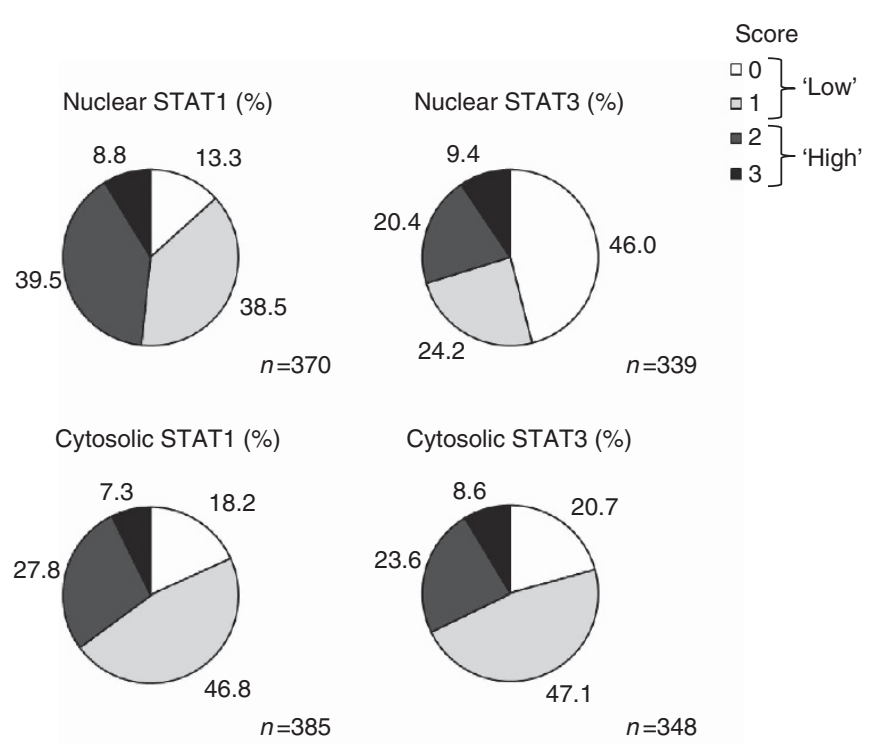

Cytosolic IL-6 (\%)

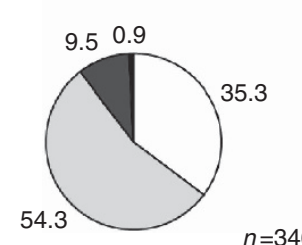

Cytosolic IL-6R $\alpha(\%)$

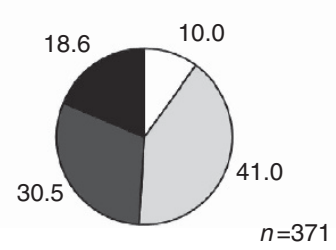

Figure 3. Immunohistochemical analysis of CRC TMAs for IL-6/STAT pathway proteins, summary of scoring results. Overall fine scoring for staining by antibodies to STATs and IL-6/IL-6R $\alpha$ and binary representation of 'low' (scores 0 and 1, light colours) vs 'high' staining intensity (scores 2 and 3, dark colours) for STATs, IL-6 and IL-6 receptor- $\alpha$.

with lymph node metastases $(P=0.094$, data not shown). Combined evaluation of the six individual parameters under study by multiple comparison employing 0.0083 as an adjusted alpha value (Bonferroni correction method) did not reach significance (data not shown).

Mutual correlation of individual STAT expression and activity in CRC biopsies. The Somer's d-test revealed significant positive mutual correlation of STAT1 and STAT3 expression (0.228; $P=0.000$ ). These results suggest that the two STAT proteins can actively influence signalling processes during tumourigenesis and cancer progression at the same time, potentially in a reciprocal manner. Furthermore, positive mutual correlation of STAT1 expression with expression of both IL-6 $(0.112 ; P=0.032)$ and the IL-6 receptor- $\alpha(0.200 ; P=0.000)$ could be shown. STAT3 expression was also positively correlated to expression of IL-6 $(0.155 ; P=0.003)$ and the IL- 6 receptor- $\alpha(0.184 ; P=0,001)$. These results are in line with the involvement of IL-6 signalling, with STAT1 and STAT3 as potential targets, which was demonstrated in CRC cell lines by us and others (Heinrich et al, 2003; Corvinus et al, 2005; Zugowski et al, 2011).

Previously, we had observed DNA-binding STAT1/3 heterodimeric complexes of STAT1 and STAT3 in both cell lines and tumour biopsies (Corvinus et al, 2005). Thus, we addressed the individual and combined activation status of STAT1 and STAT3 in a representative collection of surgical CRC specimens by electrophoretic mobility shift assay (EMSA; Figure 5A and B). In a subset of samples, the presence of STAT1 and STAT3 in complexes with a cognate DNA element was confirmed by addition of antibodies with specificity for the respective STATs to the binding reactions
(Figure 5A). Appearance of supershifted complexes revealed homo- or heterodimeric complexes of STAT1/STAT3.

Within the collection of biopsies analysed, DNA-binding activities of STAT1/3 homo- and heterodimer complexes ranged widely from very strong activities to no significant detection of STAT1/3 activity (in two samples, number 14 and number 18). Overall, STAT1/3 DNA-binding complexes are predominantly present in the vast majority of samples (20 out of 22); however, activity status and complex composition show considerable variability. We judge all significant homodimer complexes for STAT1 as an indicator for significant pYSTAT1 activity. Two samples displayed very unique STAT1 activity without significant STAT3 DNA binding (number 7 and number 20). Judgment of significant STAT3 homodimer formation is difficult as unphosphorylated STAT1 can be pulled into pYSTAT3 DNA-binding complexes (unpublished data). These findings are in line with the notion that combined activities of STAT1 and STAT3 are likely to be of functional relevance for CRC tumour cell properties. Moreover, they suggest that splicing, cleavage or post-translational modifications of STAT1/3 may exert influence on the characteristics of STAT-DNA assemblies.

STAT3 as an independent prognostic marker. For multivariate analysis, only parameters that had shown statistically significant correlation with survival in the univariate analysis were treated within a Cox proportional hazard model. The following variables were included: nuclear STAT1 (that is, activation), cytosolic STAT3 (that is, expression), lymph node status and vein invasion. A stepwise backward procedure was applied to subsequently reduce the number of variables.

All items that independently show specific influence on overall survival in multivariate survival analysis are shown in Table 2. High expression of STAT3 and presence of vein invasion proved to be statistically significant independent predictors of overall survival. Whereas the existence of vein invasion accounts for bad prognosis (hazard ratio $1.965 ; P=0.002$ ), high STAT3 expression determines a favourable prognosis for patients' overall survival. The death rate is almost divided in half (hazard ratio 0.615; $P=0.023$ ).

All other variables did not show any noticeable influence on overall survival.

\section{DISCUSSION}

This study on CRC tissue microarrays shows that expression and/ or activation of both STAT1 and STAT3 indicate a favourable clinical prognosis. Notably, STAT3 was identified as an independent predictor of disease outcome. Our data support earlier reports that identified STAT1 as a tumour suppressor whose expression and/or activation was associated with longer overall survival of cancer patients (Widschwendter et al, 2002; Simpson et al, 2010). It is still somewhat contradictory though that STAT1 on the one hand is frequently found aberrantly expressed and activated in tumour cells (in up to $44 \%$ of cases in this study), whereas on the other hand it has well-established anti-proliferative and proapoptotic effects (Kim and Lee, 2007). An explanation may be facilitated by considering combined effects of STAT1 and STAT3 on the physiology of tumour cells. Regis et al (2008) have suggested that a balance between STAT1 and STAT3 signalling in cancer cells has a crucial influence on the cells' fate, as the two transcription factors have opposing roles in the regulation of survival and proliferation.

Concerning STAT3 in relation to clinical cancer prognosis, various studies hint at tumour-promoting effects. Park et al (2008) could show that levels of activated STAT3 (pSTAT3) are significantly associated with increasing $\mathrm{T}$ - and clinical stages in 

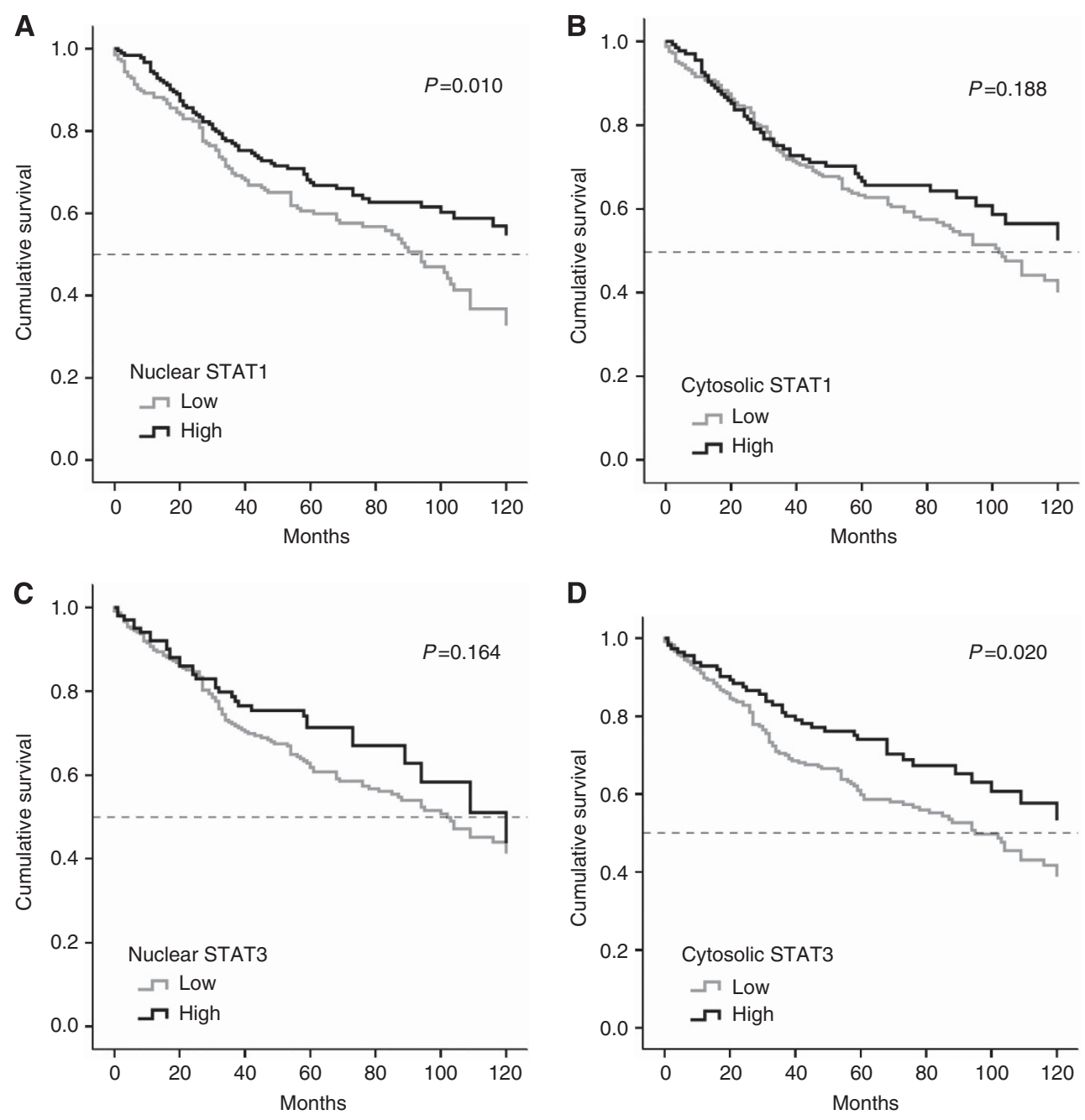

D
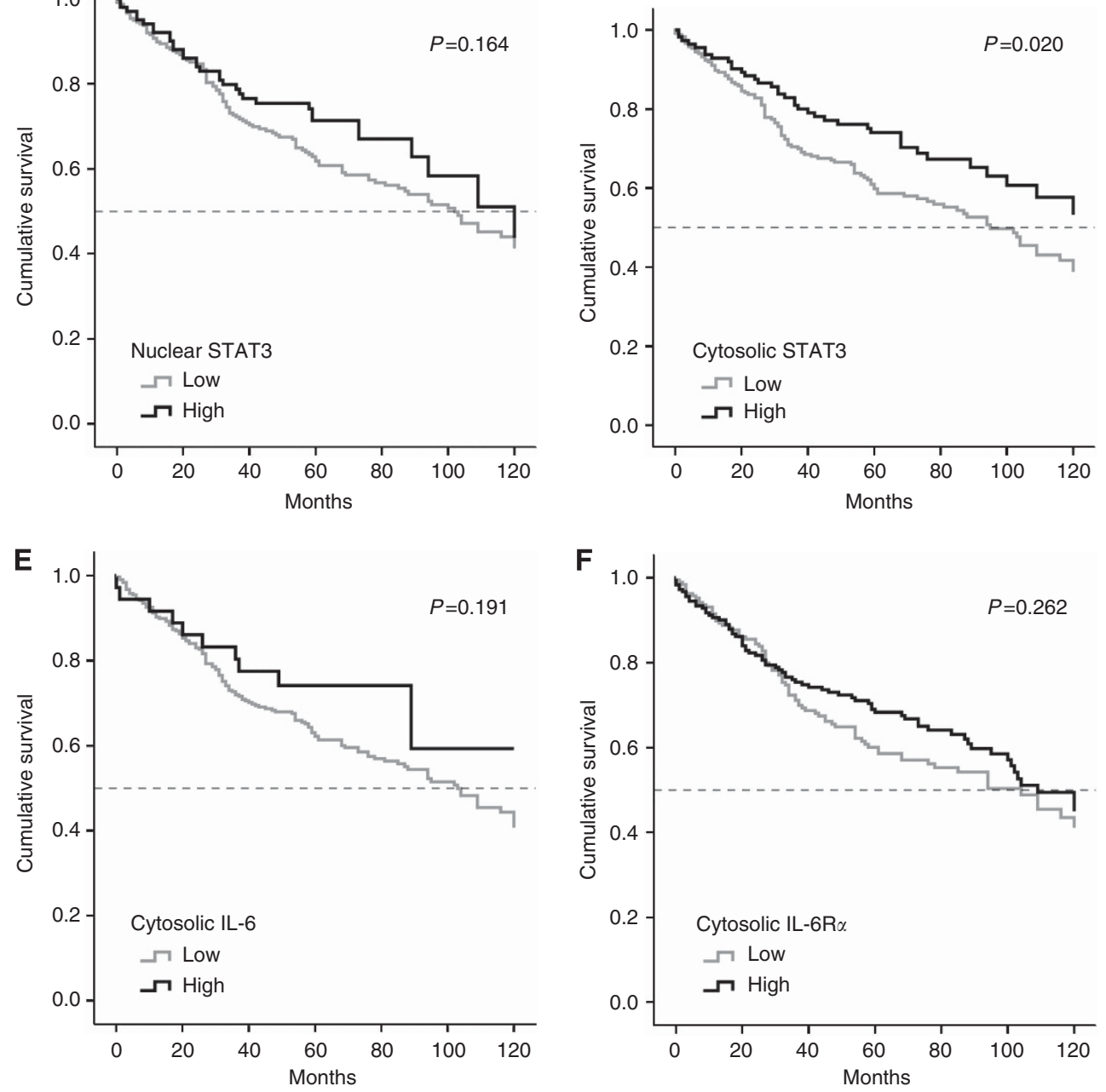

Figure 4. Separate univariate analysis of overall patients' survival in correlation to expression/activation of STATs and IL-6/IL-6R $\alpha$ in CRC TMAs. Patient survival related to (A) nuclear and (B) cytosolic STAT1, (C) nuclear and (D) cytosolic STAT3, and (E) IL-6 and (F) IL-6 receptor- $\alpha$.

CRC. Kusaba et al $(2005,2006)$ correlated pSTAT3 with increasing $\mathrm{T}$ - and Duke's stages and the presence of lymph vessel invasion, as well as with poor prognosis for overall survival in a first study, and with increasing T-stages and the presence of vein invasion and lymph node metastases in a second study of CRC. In contrast to these reports, a recent study on a French patient cohort by Monnien et al (2010) provided evidence for a correlation of
pSTAT3 appearance with prolonged survival of rectal cancer patients. An explanation for these discrepancies may lie in the largely different diet in Eastern Asia and Europe. Interestingly, all data indicating a negative role of STAT3 in disease progression were obtained with patients originating from Korea and Japan, whereas in European patients STAT3 activation appeared to be a beneficial parameter. It is well known that both diet and genetic 

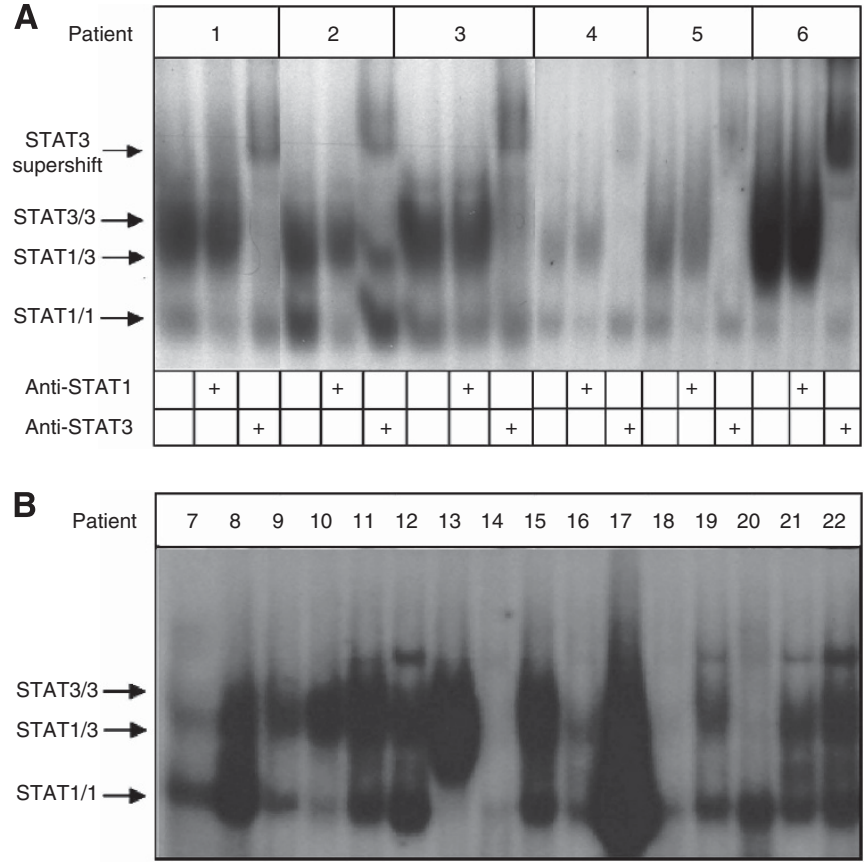

Figure 5. Analysis of the STAT activity status in an arbitrarily selected set of lysates from CRC biopsies. (A) STAT DNA-binding activity of an arbitrarily selected set of six patient samples was analysed by determining the formation of specific complexes on the high affinity AP-1 response element from the promoter of the c-fos oncogene (SIEm67). The identity of STAT-containing complexes was tested by supershift experiments employing specific antibodies to STAT1 and STAT3, as indicated. Positions of STAT1-, STAT3- and STAT1/STAT3DNA complexes are marked with arrows. (B) Analysis of a collection of further CRC biopsies for STAT DNA-binding activity. Electrophoretic mobility shift assay was performed and results are presented as in $\mathbf{A}$ except for the omission of supershift experiments.

Table 2. Prognostic CRC sample evaluation of STATs and clinical parameters in multivariate analysis of overall survival with the following variables included: nuclear STAT1, cytosolic STAT3, lymph node status and vein invasion

\begin{tabular}{|c|c|c|c|}
\hline & $\boldsymbol{P}$ & $\begin{array}{c}\text { Hazard } \\
\text { ratio }\end{array}$ & $\begin{array}{c}95 \% \\
\text { Confidence } \\
\text { interval }\end{array}$ \\
\hline \multicolumn{4}{|c|}{ STAT3 (cytosolic localisation) } \\
\hline High vs low & 0.023 & 0.615 & $0.404-0.935$ \\
\hline \multicolumn{4}{|l|}{ Vein invasion } \\
\hline Present vs absent & 0.002 & 1.965 & $1.292-2.989$ \\
\hline
\end{tabular}

background influence CRC development and progression, which could account for these discrepancies. Overall, we have employed for this tissue microarray-based study a unique and well defined, very homogeneous group of CRC patients from a local region in former East Germany. This region has a particularly high CRC incidence and more advanced CRC cases, which we viewed important to illustrate (Figure 1). Reasons for this are complex and most likely attributable to historic reasons (there was limited diagnostic medical support to detect and surgically remove, as well as to treat CRC in former East Germany). Moreover, the patient cohort included in this study can be associated with a lifestyle characterised by several risk factors such as over-average meat consumption and overweight/obesity/diabetes measures.

Our study that revealed STAT3 as a predictor of better patients' outcome in both univariate and multivariate standard survival analyses is in line with these latter results. It is particularly interesting to note that it corresponds very well to novel findings by Musteanu et al (2010) of anti-tumour effects of STAT3 in vivo, employing the $\mathrm{Apc}^{\mathrm{Min}}$ mouse model for intestinal cancer. Although multiplicity and progression of early adenomas were reduced by genetic ablation of STAT3, loss of STAT3 at later stages promoted tumour progression and favoured the development of invasive carcinomas, resulting in a significantly shorter survival. In this context, hence, absence of STAT3 was found to be a determinant of poor prognosis.

An association of STAT3 activity with favourable clinical prognosis has been reported for a number of other cancer entities such as node-negative breast cancer (Dolled-Filhart et al, 2003), nasopharyngeal cancer (Hsiao et al, 2003), prostate cancer (TorresRoca et al, 2007) and diffuse large B-cell lymphoma (Stewart et al, 2009). In contrast, STAT3 was described as indicative for poor prognosis in gastric cancer (Yakata et al, 2007) and cervical squamous-cell carcinoma (Takemoto et al, 2009).

Interestingly, activated STAT1 and STAT3 appear at the same time in the vast majority of CRC biopsies (Corvinus et al, 2005) and, as shown in this study, may interact simultaneously with STAT cognate DNA elements. This raises the possibility that activities that interfere functionally with both STAT factors, thus influencing their balance, are of importance for tumour progression.

Taken together, numerous studies have addressed the role of aberrant STAT3 function in cancer and have not yet revealed a consistent picture. On the basis of the context, STAT3 has been described as an oncogene or a tumour suppressor. Importantly, these controversial studies did so far analyse neither the relative levels of STAT1 and STAT3 abundance and activity nor the appearance of STAT1/3 assemblies. The latter presumably are interdependent DNA-binding complexes indicating mutual influences of STAT1 and STAT3, and our study is the first to take this aspect into consideration. Thus, the picture of oncogenic STAT3 needs to be viewed in a wider context and in light of STAT1 expression and activity data. In fact, our own unpublished CRC xenograft experiments demonstrate that it is the expression level of STAT1 that dictates tumour cell line growth in SCID mice (Nivarthi et al, manuscript in preparation and unpubished data). It is possible that the tumour suppressive role of STAT3 in CRC inferred from our study is a characteristic feature of the specific and particularly homogeneous patient cohort under investigation, and we cannot exclude that other patient cohorts show different properties in this respect. Genetic and environmental factors might contribute to CRC aetiology and to the functional implications of STAT activities.

Interleukin- 6 and the IL- 6 receptor $\alpha$ have been associated with proliferative and anti-apoptotic effects in tumour cells in general and in CRC in particular. Clinical and experimental data suggest a contribution of IL- 6 signalling to the development of both sporadic and colitis-associated colorectal cancer development (Atreya and Neurath, 2008). It has been shown that antibody-mediated blockade of the IL-6 receptor reduces malignant properties of human colon carcinoma cells (Hsu et al, 2011). It is interesting to note that loss of soluble IL- 6 receptor- $\alpha$, which enhances IL-6dependent cell activation via trans-signalling, was found correlated with faster progression of colorectal cancer (Okugawa et al, 2010). This is in line with our findings that link STAT1 and STAT3, both downstream mediators of IL-6 signalling, to longer survival of CRC patients. In our study, STAT1 and STAT3 expression were both 
separately found to be correlated with IL- 6 and the IL- 6 receptor- $\alpha$ chain expression, and strong expression of both IL-6 and IL-6 receptor- $\alpha$ in CRC biopsies showed a tendency for association with longer survival. In the case of IL-6, the lack of significance in survival analyses could be due to the very low frequency (10\%) of IL-6-positive tumour samples.

\section{ACKNOWLEDGEMENTS}

We thank Mario Walther for valuable advice on statistical analyses and Alexander Berndt for generous help with microscopy and image processing. This work was supported by scholarships granted to CG by the Interdisziplinäres Zentrum für klinische Forschung (IZKF) Jena and the Deutsche Krebshilfe e.V. The research project was supported by a grant from the Austrian Science Funds (FWF, SFB F28 to RM).

\section{REFERENCES}

Atreya R, Neurath MF (2008) Signaling molecules: the pathogenic role of the IL-6/STAT-3 trans signaling pathway in intestinal inflammation and in colonic cancer. Curr Drug Targets 9: 369-374.

Bromberg JF, Wrzeszczynska MH, Devgan G, Zhao Y, Pestell RG, Albanese C, Darnell Jr JE (1999) Stat3 as an oncogene. Cell 98: 295-303.

Chen Y, Knösel T, Kristiansen G, Pietas A, Garber ME, Matsuhashi S, Ozaki I, Petersen I (2003) Loss of PDCD4 expression in human lung cancer correlates with tumor progression and prognosis. J Pathol 200: 640-646.

Chung YC, Chang YF (2003) Serum interleukin-6 levels reflect the disease status of colorectal cancer. J Surg Oncol 83: 222-226.

Chung YC, Chaen YL, Hsu CP (2006) Clinical significance of tissue expression of interleukin-6 in colorectal carcinoma. Anticancer Res 26: 3905-3911.

Corvinus FM, Orth C, Moriggl R, Tsareva SA, Wagner S, Pfitzner EB, Baus D, Kaufmann R, Huber LA, Zatloukal K, Beug H, Ohlschläger P, Schütz A, Halbhuber KJ, Friedrich K (2005) Persistent STAT3 activation in colon cancer is associated with enhanced cell proliferation and tumor growth. Neoplasia 7: 545-555.

Cui T, Chen Y, Yang L, Knösel T, Zöller K, Huber O, Petersen I (2011) DSC3 expression is regulated by p53, and methylation of DSC3 DNA is a prognostic marker in human colorectal cancer. Br J Cancer 104: 1013-1019.

Dolled-Filhart M, Camp RL, Kowalski DP, Smith BL, Rimm DL (2003) Tissue microarray analysis of signal transducers and activators of transcription 3 (Stat3) and phospho-Stat3 (Tyr705) in node-negative breast cancer shows nuclear localization is associated with a better prognosis. Clin Cancer Res 9: $594-600$

Hamidov Z, Altendorf-Hofmann A, Chen Y, Settmacher U, Petersen I, Knösel T (2011) Reduced expression of desmocollin 2 is an independent prognostic biomarker for shorter patients survival in pancreatic ductal adenocarcinoma. J Clin Pathol 64: 990-994.

Heinrich PC, Behrmann I, Haan S, Hermanns HM, Müller-Newen G, Schaper F (2003) Principles of interleukin (IL)-6-type cytokine signalling and its regulation. Biochem J 374: 1-20.

Hsiao JR, Jin YT, Tsai ST, Shiau AL, Wu CL, Su WC (2003) Constitutive activation of STAT3 and STAT5 is present in the majority of nasopharyngeal carcinoma and correlates with better prognosis. Br J Cancer 89: 344-349.

Hsu CP, Chen YL, Huang CC, Chou CC, Liu CL, Hung CH, Kao TY, Chung YC (2011) Anti-interleukin-6 receptor antibody inhibits the progression in human colon carcinoma cells. Eur J Clin Invest 42: 277-284.

Kim HS, Lee MS (2007) STAT1 as a key modulator of cell death. Cell Signal 19: 454-465.

Klampfer L (2008) The role of signal transducers and activators of transcription in colon cancer. Front Biosci 13: 2888-2899.

Knösel T, Chen Y, Hotovy S, Settmacher U, Altendorf-Hofmann A, Petersen I (2012) Loss of desmocollin 1-3 and homeobox genes PITX1 and CDX2 are associated with tumor progression and survival in colorectal carcinoma. Int J Colorectal Dis 27: 1391-1399.

Komoda H, Tanaka Y, Honda M, Matsuo Y, Hazama K, Takao T (1998) Interleukin-6 levels in colorectal cancer tissues. World J Surg 22: 895-898.
Kusaba T, Nakayama T, Yamazumi K, Yakata Y, Yoshizaki A, Nagayasu T, Sekine I (2005) Expression of p-STAT3 in human colorectal adenocarcinoma and adenoma; correlation with clinicopathological factors. J Clin Pathol 58: 833-838.

Kusaba T, Nakayama T, Yamazumi K, Yakata Y, Yoshizaki A, Inoue K, Nagayasu T, Sekine I (2006) Activation of STAT3 is a marker of poor prognosis in human colorectal cancer. Oncol Rep 15: $1445-1451$.

Lee J, Kim JC, Lee SE, Quinley C, Kim H, Herdman S, Corr M, Raz E (2012) Signal transducer and activator of transcription 3 (STAT3) protein suppresses adenoma-to-carcinoma transition in Apcmin/ + mice via regulation of Snail-1 (SNAI) protein stability. J Biol Chem 287: 18182-18189.

Monnien F, Zaki H, Borg C, Mougin C, Bosset JF, Mercier M, Arbez-Gindre F, Kantelip B (2010) Prognostic value of phosphorylated STAT3 in advanced rectal cancer: a study from 104 French patients included in the EORTC 22921 trial. J Clin Pathol 63: 873-878.

Murray PJ (2007) The JAK-STAT signaling pathway: input and output integration. J Immunol 178: 2623-2629.

Musteanu M, Blaas L, Mair M, Schlederer M, Bilban M, Tauber S, Esterbauer H, Mueller M, Casanova E, Kenner L, Poli V, Eferl R (2010) Stat3 is a negative regulator of intestinal tumor progression in $\mathrm{Apc}(\mathrm{Min})$ mice. Gastroenterology 138: 1003-1011.

Okugawa Y, Miki C, Toiyama Y, Yasuda H, Yokoe T, Saigusa S, Hiro J, Tanaka K, Inoue Y, Kusunoki M (2010) Loss of tumoral expression of soluble IL-6 receptor is associated with disease progression in colorectal cancer. Br J Cancer 103: 787-795.

Park JK, Hong R, Kim KJ, Lee TB, Lim SC (2008) Significance of p-STAT3 expression in human colorectal adenocarcinoma. Oncol Rep 20: 597-604.

Regis G, Pensa S, Boselli D, Novelli F, Poli V (2008) Ups and downs: the STAT1:STAT3 seesaw of interferon and gp130 receptor signalling. Semin Cell Dev Biol 19: 351-359.

Rose-John S, Mitsuyama K, Matsumoto S, Thaiss WM, Scheller J (2009) Interleukin-6 trans-signaling and colonic cancer associated with inflammatory bowel disease. Curr Pharm Des 15: 2095-2103.

Sansone P, Bromberg J (2012) Targeting the interleukin-6/Jak/Stat pathway in human malignancies. J Clin Oncol 30: 1005-1014.

Simpson JA, Al-Attar A, Watson NF, Scholefield JH, Ilyas M, Durrant LG (2010) Intratumoral T cell infiltration, MHC class I and STAT1 as biomarkers of good prognosis in colorectal cancer. Gut 59: 926-933.

Stark GR, Darnell Jr JE (2012) The JAK-STAT pathway at twenty. Immunity 36: 503-514.

Stewart DA, Bahlis N, Mansoor A (2009) pY-STAT3 and p53 expression predict outcome for poor prognosis diffuse large B-cell lymphoma treated with high dose chemotherapy and autologous stem cell transplantation. Leuk Lymphoma 50: 1276-1782.

Takemoto S, Ushijima K, Kawano K, Yamaguchi T, Terada A, Fujiyoshi N, Nishio S, Tsuda N, Ijichi M, Kakuma T, Kage M, Hori D, Kamura T (2009) Expression of activated signal transducer and activator of transcription-3 predicts poor prognosis in cervical squamous-cell carcinoma. Br J Cancer 101: 967-972.

Tanaka T, Narazaki M, Kishimoto T (2012) Therapeutic targeting of the interleukin-6 receptor. Annu Rev Pharmacol Toxicol 52: 199-219.

Torres-Roca JF, DeSilvio M, Mora LB, Khor LY, Hammond E, Ahmad N, Jove R, Forman J, Lee RJ, Sandler H, Pollack A (2007) Activated STAT3 as a correlate of distant metastasis in prostate cancer: a secondary analysis of Radiation Therapy Oncology Group 86-10. Urology 69: 505-509.

Tsareva SA, Moriggl R, Corvinus FM, Wiederanders B, Schütz A, Kovacic B, Friedrich K (2007) Signal transducer and activator of transcription 3 activation promotes invasive growth of colon carcinomas through matrix metalloproteinase induction. Neoplasia 9: 279-291.

Widschwendter A, Tonko-Geymayer S, Welte T, Daxenbichler G, Marth C, Doppler W (2002) Prognostic significance of signal transducer and activator of transcription 1 activation in breast cancer. Clin Cancer Res 8 3065-3074.

Xiong H, Zhang ZG, Tian XQ, Sun DF, Liang QC, Zhang YJ, Lu R, Chen YX, Fang JY (2008) Inhibition of JAK1, 2/STAT3 signaling induces apoptosis, cell cycle arrest, and reduces tumor cell invasion in colorectal cancer cells. Neoplasia 10: 287-297.

Xiong H, Hong J, Du W, Lin YW, Ren LL, Wang YC, Su WY, Wang JL, Cui Y, Wang ZH, Fang JY (2012) Roles of STAT3 and ZEB1 proteins in 
E-cadherin down-regulation and human colorectal cancer epithelialmesenchymal transition. J Biol Chem 287: 5819-5832.

Yakata Y, Nakayama T, Yoshizaki A, Kusaba T, Inoue K, Sekine I (2007) Expression of p-STAT3 in human gastric carcinoma: significant correlation in tumour invasion and prognosis. Int J Oncol 30: 437-442.

Zhuang Q, Hong F, Shen A, Zheng L, Zeng J, Lin W, Chen Y, Sferra TJ, Hong Z, Peng J (2012) Pien Tze Huang inhibits tumor cell proliferation and promotes apoptosis via suppressing the STAT3 pathway in a colorectal cancer mouse model. Int J Oncol 40: 1569-1574.
Zugowski C, Lieder F, Müller A, Gasch J, Corvinus FM, Moriggl R, Friedrich K (2011) STAT3 controls matrix metalloproteinase-1 expression in colon carcinoma cells by both direct and AP-1-mediated interaction with the MMP-1 promoter. Biol Chem 392: 449-459.

This work is published under the standard license to publish agreement. After 12 months the work will become freely available and the license terms will switch to a Creative Commons AttributionNonCommercial-Share Alike 3.0 Unported License. 